\title{
Fast growth of supermassive black holes in galaxies
}

\author{
F. Munyaneza ${ }^{\star}$ and P. L. Biermann
}

\author{
Max-Planck-Institut für Radioastronomie, Auf dem Hügel 69, 53121 Bonn, Germany \\ e-mail: munyanez@mpifr-bonn.mpg.de
}

Received 29 October 2003 / Accepted 24 February 2005

\begin{abstract}
We report on a calculation of the growth of the mass of supermassive black holes at galactic centers from dark matter and Eddington - limited baryonic accretion. Assuming that dark matter halos are made of fermions and harbor compact degenerate Fermi balls of masses from $10^{3} M_{\odot}$ to $10^{6} M_{\odot}$, we find that dark matter accretion can boost the mass of seed black holes from about $\sim 5 M_{\odot}$ to $10^{3-4} M_{\odot}$ black holes, which then grow by Eddington-limited baryonic accretion to supermassive black holes of $10^{6-9} M_{\odot}$. We then show that the formation of the recently detected supermassive black hole of $3 \times 10^{9} M_{\odot}$ at a redshift of $z=6.41$ in the quasar SDSS J114816.64+525150.3 could be understood if the black hole completely consumes the degenerate Fermi ball and then grows by Eddington-limited baryonic accretion. In the context of this model we constrain the dark matter particle masses to be within the range from $12 \mathrm{keV} / c^{2}$ to about $450 \mathrm{keV} / c^{2}$. Finally we investigate the black hole growth dependence on the formation time and on the mass of the seed black hole. We find that in order to fit the observed data point of $M_{\mathrm{BH}} \sim 3 \times 10^{9} M_{\odot}$ and $z \sim 6.41$, dark matter accretion cannot start later than about $2 \times 10^{8}$ years and the seed $\mathrm{BH}$ cannot be greater than about $10^{4} M_{\odot}$. Our results are in full agreement with the WMAP observations that indicate that the first onset of star formation might have occurred at a redshift of $z \sim 15-20$. For other models of dark matter particle masses, corresponding constraints may be derived from the growth of black holes in the center of galaxies.
\end{abstract}

Key words. black holes physics - galaxies: nuclei - cosmology: dark matter - galaxies: quasars: general

\section{Introduction}

Over the past few years, the idea of dark matter (DM) and the possible existence of supermassive black holes $(\mathrm{BH})$ of masses from $10^{6.5}$ to $10^{9.5} M_{\odot}$ at the center of galaxies (Macchetto et al. 1997; Schödel et al. 2002) have become deeply rooted within the astrophysical community. The exploration of the relationship between these two intriguing problems of modern astrophysics has been the subject of an increasing number of papers.

It has been established that the mass of the central $\mathrm{BH}$ is tightly correlated with the velocity dispersion $\sigma$ of its host bulge, where it is found that $M_{\mathrm{BH}} \sim \sigma^{4-5}$ (Faber et al. 1997; Magorrian et al. 1998; Ferrarese \& Merritt 2000; Gebhardt et al. 2000; Ferrarese 2002; Haering \& Rix 2004). This tight relation between the masses of the $\mathrm{BHs}$ and the gravitational potential well that hosts them suggests that the formation and evolution of supermassive BHs and the bulge of the parent galaxy may be closely related, e.g. Wang et al. (2000). In addition, the recent discovery of high redshift quasars with $z>6$ (Fan et al. 2001) implies that the formation of supermassive BHs took place over fewer than $10^{9}$ years. In spite of the vast and tantalizing work undertaken on BHs, their genesis and evolution are not well understood (see Rees 1984, for a review).

\footnotetext{
^ Humboldt Fellow.
}

Since the discovery of quasars in the early 1960s, it has been suggested that these objects are powered by accretion of gas onto the supermassive BHs of masses $10^{6}-10^{9} M_{\odot}$ (Lynden-Bell 1969). Two scenarios have been discussed in modeling the growth of BHs. One is that BHs grow out of a low mass "seed" BH through accretion (Rees 1984), and another one is that BHs grow by merging (Barkana et al. 2001; Wang et al. 2000; Gopal-Krishna et al. 2003, 2004). In a recent paper, Duschl \& Strittmatter (2004) have investigated a model for the formation of supermassive black holes using a combination of merging and accretion mechanisms.

The purpose of this paper is to study the growth of BHs from dark matter and Eddington-limited baryonic accretion. Also, we would like to obtain some constraints on the DM particle masses. In the past, self gravitating neutrino matter has been suggested as a model for quasars, with neutrino masses in the range $0.2 \mathrm{keV} \lesssim m_{\mathrm{f}} \lesssim 0.5 \mathrm{MeV}$ (Markov 1964). Later, neutrino matter was suggested to describe DM in clusters of galaxies and galactic halos with masses in the range of $1 \mathrm{eV} \lesssim m_{\mathrm{f}} \lesssim 25 \mathrm{eV}$ (Cowsick \& McClelland 1973; Ruffini 1980). More recently, fermion balls (FBs) made of degenerate fermionic matter of $10 \mathrm{keV} \lesssim m_{\mathrm{f}} \lesssim 25 \mathrm{keV}$ were suggested as an alternative to supermassive BHs in galaxies (Viollier 1994; Bilić et al. 1999; Tsiklauri \& Viollier 1998; Munyaneza \& Viollier 2002). It has been also suggested that if the Galaxy 
harbors a supermassive $\mathrm{BH}$, then there should be a density spike in which dark matter (DM) falling towards the center could annihilate and the detection of these annihilation signals could be used as a probe for the nature of DM (Bertone et al. 2002; Gondolo \& Silk 1999; and Merritt et al. 2002). The current belief is that DM particles are bosonic and very massive, i.e. $m_{\mathrm{DM}} \gtrsim 1 \mathrm{GeV} / c^{2}$. However, the absence of experimental constraints on the weakly interacting massive particles (WIMPs) that probably constitute DM leaves the door open for further investigation of the hidden mass of the Universe.

In this paper, we will assume DM to be of fermionic matter and described by a Fermi-Dirac distribution with an energy cutoff in phase space (King 1966). We will then explore the limits for the DM particle masses in order to reproduce the mass distribution in the Galaxy (Wilkinson \& Evans 1999) and then study the growth of a seed BH immersed at the center of the DM distribution in galaxies. We use degenerate FBs at the center of DM halos not as replacements for the BHs but as necessary ingredients to grow the BHs in galactic centers.

The resulting distribution of stars around a massive BH was studied in detail in the 1970s and early 1980s in the context of globular clusters (Hills 1975; Frank \& Rees 1976; Bahcall \& Wolf 1976; Duncan \& Shapiro 1982; Shapiro 1985). Peebles (1972) studied the adiabatic growth of a BH in an isothermal sphere and showed that the $\mathrm{BH}$ would alter the matter density to an adiabatic cusp with $\rho \sim r^{-3 / 2}$. A few years later, Young (1980) constructed numerical models that confirmed Peebles' results and showed that the $\mathrm{BH}$ induces a tangential anisotropy in the velocity dispersion. In this paper, we use the results of previous calculations on the growth of BHs that are accreting stars (Lightman \& Shapiro 1978) to investigate the growth of a $\mathrm{BH}$ that accretes DM. Here we assume that the physics driving the formation of the power law cusp in the star - star case is the same as in the case of DM particle orbits being perturbed by molecular clouds. Julian (1967) investigated a similar scenario in which the stellar orbits in our Galaxy were perturbed by molecular clouds to explain the stellar velocity dispersion dependence on the star's age. Moreover, Duncan \& Wheeler (1980) investigated the anisotropy of the velocity dispersions of the stars around the BH in M87.

Given the density distribution in DM halos, we are interested in establishing how a seed $\mathrm{BH}$ would grow by accreting DM. Moreover, the comparison of the growth of the $\mathrm{BH}$ from accretion of DM and Eddington-limited baryonic matter would give us another piece of information in the debate surrounding the nature of DM. We therefore investigate how a $\mathrm{BH}$ seed of $5 M_{\odot}$ could grow to a $3 \times 10^{9} M_{\odot} \mathrm{BH}$ as recently detected in quasar SDSS J1148+5251 at $z=6.41$ (Willot et al. 2003). Such a seed $\mathrm{BH}$ of a typical mass between 5 and $9 M_{\odot}$ could have evolved in BH binaries (Podsiadlowski et al. 2003). Here, we note that the seed $\mathrm{BH}$ could be an intermediate mass black hole (IMBH) of $10^{3-4} M_{\odot}$ that might have formed from collisions in dense star-forming regions (Portegies Zwart \& McMillan 2002; Coleman Miller 2003; and van der Marel 2003, for a review). In fact, Wang \& Biermann (1998) have established that a BH would grow exponentially with time accreting baryonic matter as long as the supply lasts. In addition, they were able to reproduce the observed correlation $M_{\mathrm{BH}} / M_{\mathrm{sph}}$ using standard disk galaxy parameters. Assuming a cusp - like distribution of self-interacting DM (Spergel \& Steinhardt 2000), Ostriker (2000) has estimated that BHs could grow to $10^{6}-10^{9} M_{\odot}$ from $\mathrm{DM}$ accretion. For completeness, we note that accretion of DM particles by BHs has recently been studied in Zhao et al. (2002) and Read \& Gilmore (2003).

Cosmological parameters of $H_{0}=70 \mathrm{~km} \mathrm{~s}^{-1} \mathrm{Mpc}^{-1}, \Omega_{\mathrm{m}}=$ 0.3 and $\Omega_{\Lambda}=0.7$ are assumed throughout this paper. In Sect. 2, we establish the main equations to describe DM in galaxies. We then discuss the growth of seed BHs from DM and Eddingtonlimited baryonic matter accretion in Sect. 3 and conclude with a discussion in Sect. 4.

\section{Dark matter in galaxies}

\subsection{Main equations}

We characterize DM by its mass density $\rho(r)$ and its velocity dispersion $\sigma$. DM is assumed to be collisionless and of fermionic matter and the mass of DM particles is denoted by $m_{\mathrm{f}}$. We will look for DM distributions with degenerate cores at the center of the DM halos instead of a steep power law $\rho \sim r^{-\gamma}$ with $\gamma \approx 1-1.5$ (e.g. Navarro et al. 1997) as we are assuming here that a central cusp is a result of BH growth. In order to obtain bound astrophysical solutions at finite temperature, we follow King (1966) and introduce an energy cutoff in phase space using the Fermi-Dirac distribution function. We therefore adopt the following prescription for the distribution function

$f(E)= \begin{cases}\frac{g_{\mathrm{f}}}{8 \pi^{3} \hbar^{3}} \frac{1}{\exp \left(\frac{E-\mu}{k T}\right)+1}+\mathrm{C}, & E<E_{\mathrm{c}} \\ 0, & E>E_{\mathrm{c}},\end{cases}$

where $g_{\mathrm{f}}$ is the spin degree of freedom, i.e. $g_{\mathrm{f}}=2$ for Majorana and $g_{\mathrm{f}}=4$ for Dirac's fermions, $\hbar$ is the Planck constant, $E$ is the particle energy, $\mu$ is the chemical potential and $T$ stands for temperature. The constant $C$ is chosen so that the distribution function vanishes at the cutoff energy $E=E_{\mathrm{c}}$. As a result, we get the following "lowered" Fermi-Dirac distribution that will be used throughout the paper.

$f\left(E, E_{\mathrm{c}}\right)= \begin{cases}\frac{g_{\mathrm{f}}}{8 \pi^{3} \hbar^{3}} \frac{\exp \left(\frac{E_{\mathrm{c}}-\mu}{k T}\right)}{\exp \left(\frac{E_{\mathrm{c}}-}{k T}\right)+1} \times \frac{1-\exp \left(\frac{E-E_{\mathrm{c}}}{k T}\right)}{\exp \left(\frac{E-\mu}{k T}\right)+1}, & E<E_{\mathrm{c}} \\ 0, & E>E_{\mathrm{c}} .\end{cases}$

The mass density $\rho(r)$ is given by

$\rho(r)=m_{\mathrm{f}} \int_{0}^{E_{\mathrm{c}}} 4 \pi f(p) p^{2} \mathrm{~d} p(E)$,

and the gravitational potential $\Phi$ obeys Poisson's equation

$\Delta \Phi(r)=4 \pi G \rho(r)$.

Next we introduce a dimensionless gravitational potential $v$, a radial variable $x$ and an inverse temperature $\beta$ as

$v=\frac{r\left(\Phi_{0}-\Phi(r)\right)}{G M_{\odot}}, \quad x=\frac{r}{a}, \beta=\frac{T_{0}}{T}$, 
where $a$ and $T_{0}$ are the length and temperature scales, respectively. They are given as

$a=\left[\frac{3 \pi \hbar^{3}}{2^{5 / 2} m_{\mathrm{f}}^{4} g_{\mathrm{f}} G^{3 / 2} M_{\odot}^{1 / 2}}\right]^{2 / 3}, \quad T_{0}=\frac{G M_{\odot} m_{\mathrm{f}}}{a k}$,

where $k$ is Boltzmann's constant and the cutoff energy $E_{\mathrm{c}}$ has been chosen as $E_{\mathrm{c}}=m_{\mathrm{f}} \Phi_{0}$. We also define the degeneracy parameter $\Lambda$ as

$\Lambda=\exp \left(\frac{m_{\mathrm{f}} \Phi_{0}-\mu}{k T}\right)$

The equation for the gravitational potential can thus be written as

$\frac{1}{x} \frac{\mathrm{d}^{2} v}{\mathrm{~d} x^{2}}=-\frac{3}{2} \beta^{-3 / 2} \frac{\Lambda}{\Lambda+1} I_{1 / 2}\left(\Lambda, \frac{v \beta}{x}\right)$,

with boundary conditions

$v(0)=\frac{M_{\mathrm{BH}}}{M_{\odot}}, \quad v\left(x_{0}\right)=0$,

where $x_{0}=R / a$ is the dimensionless size of the DM halo at which the density and pressure of DM vanishes. It can be shown that for $\Lambda \rightarrow \infty$, Eq. (8) describes the isothermal gas sphere of King (1966) whereas for $\Lambda \rightarrow \infty$, and $v \gg 1$ we recover the Lane-Emden equation for a degenerate FB (Viollier 1994). $I_{n}(k, \eta)$ is a Fermi-like integral defined as

$I_{n}(\Lambda, \eta)=\int_{0}^{\eta} \mathrm{d} \xi \xi^{n} \frac{1-\mathrm{e}^{\xi-\eta}}{1+\Lambda \mathrm{e}^{\xi-\eta}}$.

The mass density $\rho(r)$ can be written in terms of the dimensionless potential $v$ as follows:

$\rho(r)=\frac{m_{\mathrm{f}}^{4} g_{\mathrm{f}} 2^{1 / 2}}{2 \pi^{2} \hbar^{3}}\left(\frac{G M_{\odot}}{a}\right)^{3 / 2} \beta^{-3 / 2} \frac{\Lambda}{\Lambda+1} I_{1 / 2}\left(\Lambda, \frac{\beta v}{x}\right)$.

Using $\rho(r)$ and the differential equation for the gravitational potential $v$, the mass enclosed within a radius $r$ of the DM halo is given by

$$
\begin{aligned}
M(r) & =\int_{0}^{r} 4 \pi \rho\left(r^{\prime}\right) r^{\prime 2} \mathrm{~d} r^{\prime} \\
& =M_{\odot}\left(-x v^{\prime}(x)+v(x)-v(0)\right),
\end{aligned}
$$

and the total mass of the DM halo (including $M_{\mathrm{BH}}$ and the fermion halo) is given by

$M=M(R)=-M_{\odot} v^{\prime}\left(x_{o}\right) x_{0}$,

where $x_{0}$ is the dimensionless size of the DM halo. We then study the solutions of Eq. (8) for various values of $\Lambda$ and $m_{\mathrm{f}}$.

In Fig. 1, we plot the mass density and the mass enclosed for solutions with a $3 \times 10^{12} M_{\odot}$ mass and a radius of $200 \mathrm{kpc}$, which corresponds to our Galaxy. Throughout our paper, we will study solutions of type 3 which have degenerate FBs in the center. For the case of our galaxy, in order to have a degenerate FB of $3 \times 10^{6} M_{\odot}$ at the center and fit the rotation curve of the Galaxy, i.e. reproduce the $1 / r^{2}$ law, the mass of fermions should be about $m_{\mathrm{f}} c^{2} \sim 12 \mathrm{keV}$. For a FB of $3 \times$ $10^{3} M_{\odot}$, one would need a fermion mass of $m_{\mathrm{f}} \sim 450 \mathrm{keV} / c^{2}$.

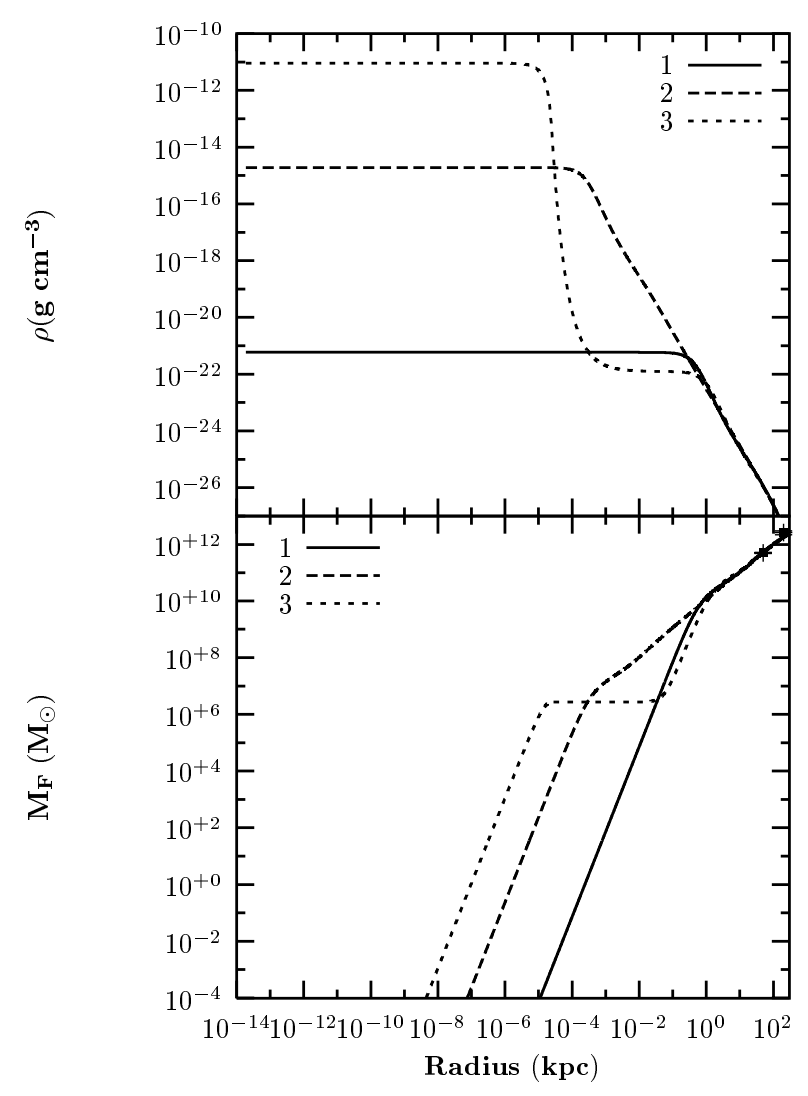

Fig. 1. The mass density and the mass enclosed within a radius $r$ are plotted in the upper and lower panels, respectively. Near the center of the DM halo, the fermions are completely degenerate and condensed in a FB of mass $\sim 3 \times 10^{6} M_{\odot}$. In the outer edge of the DM halo, the density $\rho$ scales as $1 / r^{2}$ (van Albada et al. 1985). The mass of fermions used in this plot is $m_{\mathrm{f}} c^{2} \approx 12 \mathrm{keV}$ and the size of the FB is about $10^{-2} \mathrm{pc}$. The data points for the mass within $50 \mathrm{kpc}$ and $200 \mathrm{kpc}$ are taken from Wilkinson \& Evans (1999).

Thus under the assumption of a degenerate FB of $3 \times 10^{3} M_{\odot}$ to $3 \times 10^{6} M_{\odot}$ at the center of a DM halo of $3 \times 10^{12} M_{\odot}$ with a density scaling as $1 / r^{2}$ in the outer edge of the halo, the fermion mass is constrained in the range

$12 \mathrm{keV} \lesssim m_{\mathrm{f}} c^{2} \lesssim 450 \mathrm{keV}$.

Similar solutions for the DM distribution in our Galaxy have also been studied in Bilić et al. (2002a).

The solutions shown in Fig. 1 have been obtained using the fact that at large distances the Fermi gas is non degenerate. The degree of non-degeneracy is described by Eq. (7) which contains the chemical potential $\mu$. Thus, the use of the chemical potential in the Fermi-Dirac ditribution allows to constrain the DM particles in the range from 12 to $450 \mathrm{keV}$, much below the Lee-Weinberg lower limit of about $2 \mathrm{GeV}$ (Lee \& Weinberg 1977) which was established under the assumptions that DM particles were in thermodynamic equilibrium at the freeze - out temperature. We also note that a suitable candidate for the DM particle as defined by the range given in Eq. (14) could be either the gravitino, postulated in supergravity theories with a mass in the $\sim 1 \mathrm{keV} / c^{2}$ to $\sim 100 \mathrm{GeV} / c^{2}$ range (Lyth 2000 ), or the axino, with a mass in the range between $\sim 10$ and $\sim 100 \mathrm{keV} / c^{2}$, as predicted by supersymmetric extensions of the Peccei-Quinn 
solution to the strong CP problem (Goto \& Yamaguchi 1992). For a recent review on DM particle candidates, the reader is referred to Bertone et al. (2005) and Baltz (2004).

\subsection{Black hole accretion rate and Pauli principle}

We define the local dynamical (orbital) time scale and the mass accretion flow as

$t_{\mathrm{dyn}}=\frac{r}{v_{\mathrm{ff}}}$

$\dot{M}_{\mathrm{BH}}=4 \pi r^{2} \rho(r) v_{\mathrm{ff}}$,

where $v_{\mathrm{ff}}$ is the free-fall velocity

$v_{\mathrm{ff}}=\left(\frac{2 G m(r)}{r}\right)^{1 / 2}$,

and $m(r)$ is the mass enclosed within a radius $r$ in the DM halo.

Near the center of the DM halo, the core becomes completely degenerate and the Pauli condition can be written as

$\left(\frac{g_{\mathrm{f}}}{6 \pi^{2}}\right)^{1 / 3} \frac{m_{\mathrm{f}} v}{n^{1 / 3}}=\hbar$

where $n=\rho / m_{\mathrm{f}}$ is the number density for DM particles and $v$ is the Fermi velocity. A seed $\mathrm{BH}$ of mass $M_{\mathrm{BH}}$ that settles at the center of the DM halo induces a free-fall velocity

$v_{\mathrm{ff}}=\sqrt{\frac{2 G M_{\mathrm{BH}}}{r}}$.

Replacing $v$ from the Eq. (18) with the last expression for $v_{\mathrm{ff}}$, the density of DM at the Pauli limit is given by

$n=\frac{g_{\mathrm{f}}}{6 \pi^{2}} \frac{m_{\mathrm{f}}^{3}}{\hbar^{3}}\left(\frac{2 G M_{\mathrm{BH}}}{r}\right)^{3 / 2}$.

We then rewrite Eq. (16) for the mass flow using the Pauli flow condition near the $\mathrm{BH}$

$$
\begin{aligned}
\dot{M}_{\mathrm{BH}} & =\frac{8 g_{\mathrm{f}}}{3 \pi \hbar^{3}} m_{\mathrm{f}}^{4} G^{2} M_{\mathrm{BH}}^{2} \\
& =1.03 \times 10^{-7} g_{\mathrm{f}}\left(\frac{m_{\mathrm{f}} c^{2}}{15 \mathrm{keV}}\right)^{4}\left(\frac{M_{\mathrm{BH}}}{M_{\odot}}\right)^{2} M_{\odot} \mathrm{yr}^{-1}
\end{aligned}
$$

Thus as soon as the region of $\mathrm{BH}$ influence is reached, the accretion flow becomes independent of the radius. However, the $\mathrm{BH}$ accretion rate depends on the fourth power of the fermion mass and on the square of the BH mass. This dependence is of course a consequence of the Pauli principle applied near the $\mathrm{BH}$.

Here we note that the mass $M_{\mathrm{F}}$ of nonrelativistic degenerate FBs scales with radius $R_{\mathrm{F}}$ as

$M_{\mathrm{F}} R_{\mathrm{F}}^{3}=27.836 M_{\odot}\left(\frac{15 \mathrm{keV}}{m_{\mathrm{f}} c^{2}}\right)^{8}\left(\frac{2}{g_{\mathrm{f}}}\right)^{2} \mathrm{pc}^{3}$.

The physics of degenerate FBs as a model of DM at the center of galaxies was considered in a series of papers (Bilić et al. 2002a,b; Bilić et al. 1999; Munyaneza et al. 1998, 1999; Munyaneza \& Viollier 2002; Tsiklauri \& Viollier 1998;

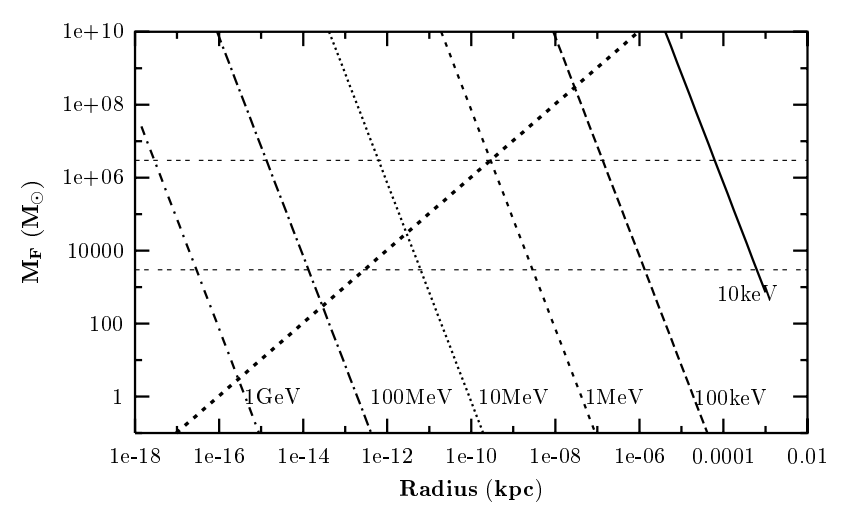

Fig. 2. The mass-radius relation for degenerate FBs. The total mass scales as $M_{\mathrm{F}} \sim R_{\mathrm{F}}^{-3} m_{\mathrm{f}}^{-8}$. In the same plot, we have shown the $\mathrm{BH}$ line and two horizontal lines for the lower and upper limits for the total mass of the degenerate Fermi balls. Relativistic effects make the massradius relation curve have a maximum mass $M_{\mathrm{OV}}$ (see Eq. (24)) and the size of such a FB at the Oppenheimer Volkoff limit is only $4.45 R_{\mathrm{OV}}^{\mathrm{s}}$ where $R_{\mathrm{OV}}^{\mathrm{s}}$ is the Schwarzchild radius of the mass $M_{\mathrm{OV}}$. The line of $\mathrm{BHs}$ and a FB at the OV limit are nearly indistinguishable in this plot.

Viollier 1994). In these papers, it was argued that the supermassive compact objects in galactic centers are FBs rather than BHs. Our approach in this paper is that FBs are not a replacement for BHs but are a necessary step for them to grow in galactic centers.

In Fig. 2 we plot the mass-radius relation for FBs for different values of the fermion mass. In the same plot we have drawn the $\mathrm{BH}$ line and two horizontal lines that correspond to a mass of $3 \times 10^{3} M_{\odot}$ and $3 \times 10^{6} M_{\odot}$. From this plot, we find an upper limit for the fermion mass of

$m_{\mathrm{f}} c^{2}<32 \mathrm{MeV}$.

This upper limit is the value of the fermion mass that would correspond to the point in the graph where the $\mathrm{BH}$ line crosses the horizontal line of a mass $M_{\mathrm{F}}=3 \times 10^{3} M_{\odot}$.

The choice of the lowest value for the FB mass is motivated by the fact that once the $\mathrm{BH}$ has consumed a FB of $3 \times 10^{3} M_{\odot}$, it would grow by Eddington-limited baryonic matter accretion to $3 \times 10^{9} M_{\odot}$ as observed in quasars at redshifts $z \sim 6$. The choice of the second value of $3 \times 10^{6} M_{\odot}$, is due to increasing evidence for the existence of a $\mathrm{BH}$ of mass $3 \times 10^{6} M_{\odot}$ at the center of our galaxy. Thus we will assume throughout this paper that galaxies which have BHs had degenerate FBs of masses ranging from $\sim 10^{3} M_{\odot}$ to $\sim 10^{6} M_{\odot}$. The fermion mass range given by Eq. (14) is of course well below the upper limit for the fermion mass from Eq. (23).

For completeness we note here that stable degenerate FBs exist up to a maximum mass also called the Oppenheimer Volkoff limit

$$
\begin{aligned}
M_{\mathrm{OV}} & =0.54195 M_{\mathrm{Pl}}^{3} m_{\mathrm{f}}^{-2} g_{\mathrm{f}}^{-1 / 2} \\
& =2.7821 \times 10^{9} M_{\odot}\left(\frac{15 \mathrm{keV}}{m_{\mathrm{f}} c^{2}}\right)^{2}\left(\frac{2}{g_{\mathrm{f}}}\right)^{2},
\end{aligned}
$$

where $M_{\mathrm{Pl}}=(\hbar c / G)^{1 / 2}$ is the Planck mass (Bilić et al. 1999). The size of a such FB at the Oppenheimer Volkoff limit is only 


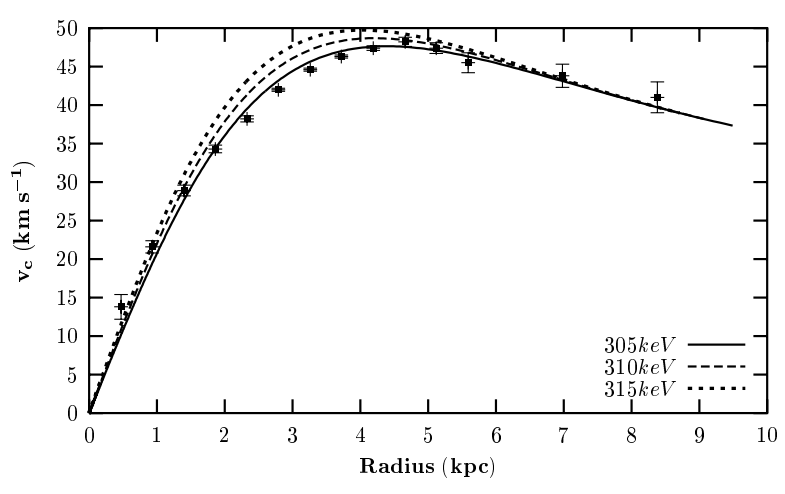

Fig. 3. Fit of the rotation curve of dwarf galaxies DD0154. The data points can be fitted by a non-degenerate Fermi-Dirac distribution function for DM particles in the mass range discussed in this paper, i.e. for $12 \mathrm{keV} \lesssim m_{\mathrm{f}} c^{2} \lesssim 450 \mathrm{keV}$. In this plot we have used a fermion mass of $m_{\mathrm{f}} \sim 305 \mathrm{keV} / c^{2}$ to fit the rotation curve of the dwarf galaxy DD0154. Here we also note that some small addition of baryonic matter would probably allow a closer fit to the data.

$R_{\mathrm{OV}}=4.45 R_{\mathrm{OV}}^{\mathrm{s}}$ where $R_{\mathrm{OV}}^{\mathrm{s}}$ is the Schwarzschild radius of the mass $M_{\mathrm{OV}}$. Thus there is practically no difference between a black hole and a FB of the same mass at the OV limit, as the closest stable orbit around a non-rotating $\mathrm{BH}$ has a radius of $3 R_{\mathrm{s}}$ only.

From Eq. (24), the fermion mass needed to form a degenerate FB of $3 \times 10^{9} M_{\odot}$ at the OV limit is about $m_{\mathrm{f}} \sim 12 \mathrm{keV} / c^{2}$. This value is in agreement with the lower limit on the mass of fermions obtained from fitting the Galactic DM halo with a FB of $3 \times 10^{6} M_{\odot}$ at the center and $1 / r^{2}$ density law from about $3 \mathrm{kpc}$. The most compact supermassive object of $3 \times 10^{9} M_{\odot}$ has been identified as a BH at the center of M87 (Macchetto et al. 1997).

Apart from fermions as candidates of DM, bosons have also been considered as an alternative to the DM constituents in galaxies. Self-gravitating bosons can form boson stars and they are prevented from complete gravitational collapse by the Heisenberg uncertainty principle $\Delta r \Delta p \sim \hbar$ (Schunck \& Liddle 1977). Boson stars are described by Einstein's field equations coupled with the non-linear Klein Gordon equation for a complex field $\Psi$ with self-interaction of the form $U(|\Psi|)=m_{\mathrm{B}}^{2}|\Psi|^{2}+\kappa|\Psi|^{4}$ where $m_{\mathrm{B}}$ is the boson mass and $\kappa$ is a coupling constant. It has been shown that boson stars have a maximum mass called the Kaup limit given by

$M_{\text {Kaup }}=0.633 M_{\mathrm{Pl}}^{2} / m_{\mathrm{B}}$,

for $\kappa=0$. Thus for a boson mass of $m_{\mathrm{B}} \sim 10^{-23} \mathrm{GeV} / c^{2}$ the Kaup mass limit is about $10^{6} M_{\odot}$ (Torres et al. 2000). For $m_{\mathrm{B}} \sim$ $1 \mathrm{GeV} / c^{2}$ the Kaup limit is as small as $10^{-17} M_{\odot}$. However if $\kappa \neq 0$, the combination of these two free parameters $m_{\mathrm{B}}$ and $\kappa$ let boson stars have any mass for any chosen value of the boson mass $m_{\mathrm{B}}$. It is in this framework that boson stars were attractive as they could form very massive objects with $m_{\mathrm{B}} \geq 1 \mathrm{GeV} / c^{2}$ (Schunck \& Mieke 2003).

In Fig. 3, we fit the rotation curve of dwarf galaxies using a Fermi-Dirac distribution. The rotation curve data points are well fitted in the outer region. In the inner region, i.e. around $1 \mathrm{kpc}$, baryonic matter is perhaps needed to get a better fit of the rotation curve. In order to fit the rotation curve of dwarf galaxies, a non-degenerate FD distribution is needed. The data points are taken from Carignan \& Purton (1998) and the mass of the dwarf galaxy DD0154 is taken to be $3 \times 10^{9} M_{\odot}$ with a size of $\sim 10 \mathrm{kpc}$ (Kravtsov et al. 1998). The merging of dwarf galaxies would generate another galaxy with a degenerate FB, and the $\mathrm{BH}$ would grow by the same mechanism considered in this paper in the merged galaxy. The Fermi-Dirac distribution studied in this paper allows two types of solutions: one which fits dwarf galaxies with a non-degenerate core and the other solution with $1 / r^{2}$ drop off in the outer edge of the halo. Degenerate FBs are consistent with data on the rotational curves only in the second type of solution with a $1 / r^{2}$ density profile at large distances.

\section{Black hole growth}

\subsection{Inner dark matter accretion and the quantum cascade mechanism}

In this section we will consider the accretion of inner DM, i.e. DM inside the FB and in the next section we will analyse the accretion of DM outside the FB. In the last section, we established that the inner part of the the Fermi ball is completely degenerate and have used the Pauli Principle to obtain the $\mathrm{BH}$ consumption rate given by Eq. (21), which can be integrated to give the $\mathrm{BH}$ mass as a function of time:

$M_{\mathrm{BH}}=M_{\odot}\left[\frac{M_{\odot}}{M_{\mathrm{BH}}^{i}}-\frac{1}{t_{0}}\left(t-t^{i}\right)\right]^{-1}$,

where $M_{\mathrm{BH}}^{i}$ is the mass of the seed BH at the starting time $t^{i}$ of accretion, and $t_{0}$ is a time parameter given by

$t_{0}=9.7 \times 10^{6} \mathrm{yr}\left(\frac{15 \mathrm{keV}}{m_{\mathrm{f}} c^{2}}\right)^{4} g_{\mathrm{f}}^{-1}$

The last two equations define the growth of a seed $\mathrm{BH}$ immersed at the center of a DM halo with a degenerate FB from which it is seen that the $\mathrm{BH}$ mass strongly depends on the fermion mass.

From Eq. (26), we can estimate the time $t_{\infty}$ needed for the $\mathrm{BH}$ mass to be infinite to be

$t_{\infty}=t^{i}+\frac{M_{\odot}}{M_{\mathrm{BH}}^{i}} t_{0}$,

which for an initial seed of $5 M_{\odot}$ at $t^{i}=0$ would give

$t_{\infty}=1.94 \times 10^{6} \mathrm{yr}\left(\frac{15 \mathrm{keV}}{m_{\mathrm{f}} c^{2}}\right)^{4} g_{\mathrm{f}}^{-1}$

For a fermion mass of $m_{\mathrm{f}}=1 \mathrm{keV} / c^{2}$ the above timescale $t_{\infty}$ would be about $5 \times 10^{10}$ years whereas a fermion mass of $m_{\mathrm{f}}=1 \mathrm{MeV} / c^{2}$ would give a growth timescale of about $t_{\infty}=5 \times 10^{-2}$ years. However, the BH mass cannot be greater than the mass of the FB that feeds it and we will require that the BH mass will grow from DM accretion only up to the mass of the FB and this will constitute one of the main assumptions in this paper. Once the BH mass has grown to the FB ball mass, 
a further growth to $10^{6-9} M_{\odot}$ is achieved via Eddington-limited baryonic matter accretion.

The mechanism of consumption of DM particles by the $\mathrm{BH}$ can be understood using a quantum cascade mechanism. First, low angular momentum DM particles at the inner Fermi surface in phase space will be consumed by the $\mathrm{BH}$. Due to a high degeneracy pressure, high angular momentum particles will be pushed to the inner Fermi surface and taken up by the BH. Outside the FB, fermions will hit the outer Fermi surface and for the FB to be hit continuously by DM particles, we use molecular clouds as perturbers of DM orbits. Thus, we have a three body interaction between the FB, the molecular clouds and DM particles. For our mechanism of consumption of DM by the BH to work, we will assume that the dark matter particles interact with the FB through inelastic collisions. In hitting the FB, some of the DM particles will be excited to a slightly higher energy level and get stuck in high momentum segments of phase space. This process will continue until the FB has been completely consumed by the $\mathrm{BH}$ and thereafter baryonic matter accretion will control the $\mathrm{BH}$ growth.

\subsection{Outer dark matter accretion}

We study the growth of a seed $\mathrm{BH}$ of about $5 M_{\odot}$ introduced at the center of DM halos of masses ranging from $10^{9} M_{\odot}$ to $10^{14.5} M_{\odot}$. The lower limit corresponds to dwarf galaxy halo masses while the upper limit corresponds to the most massive halo such as that of M87. The gravitational potential and the mass density of DM are given by Eqs. (8) and (11), respectively. It is well established that the $\mathrm{BH}$ will induce a density law $\rho(r) \sim r^{-3 / 2}$ at the center of a DM halo (Lightman \& Shapiro 1978; Quinlan et al. 1995; MacMillan \& Henriksen 2002; Merritt 2003). Following Spitzer \& Schwarzschild (1951, 1953) and Julian (1967), we assume that DM orbits outside the degenerate FB are perturbed by molecular clouds of mass $m_{\text {pert }} \approx 10^{6} M_{\odot}$. It has been recently reported that molecular gas of total mass $\sim 10^{10} M_{\odot}$ is observed in the host galaxy of the same quasar SDSS $\mathrm{J} 114816.64+525150.3$ at $z \approx 6.41$ (Walter et al. 2003; Carilli et al. 2006).

In this paper, we assume that there is a compact degenerate $\mathrm{FB}$ at the center of galaxies that have $\mathrm{BHs}$. The seed $\mathrm{BH}$ first consumes the degenerate FB and grows to a mass of about $10^{3-6} M_{\odot}$ and then grows by Eddington-limited baryonic matter accretion to higher masses of $10^{6-9} M_{\odot}$.

We will be mostly concerned with the calculation of the time needed to refill the $\mathrm{BH}$ loss cone using molecular clouds as DM perturbers. To this effect, we denote by $r$ the radial distance from the $\mathrm{BH}$ to the $\mathrm{DM}$ particle position, $\tilde{r}$ the distance from the $\mathrm{BH}$ to the molecular clouds, $\theta$ is the angle between the direction from the $\mathrm{BH}$ to the $\mathrm{DM}$ particle and its projection in the disk and $\varphi$ is the angle between the direction from the $\mathrm{BH}$ to the molecular clouds and to the foot of the DM particle location in the disk. The diagram for the geometry used is shown in Fig. 4.

In order to get the expression for the time $\tau_{\text {refill }}(r)$ needed to refill the loss cone which is due to multiple small-angle

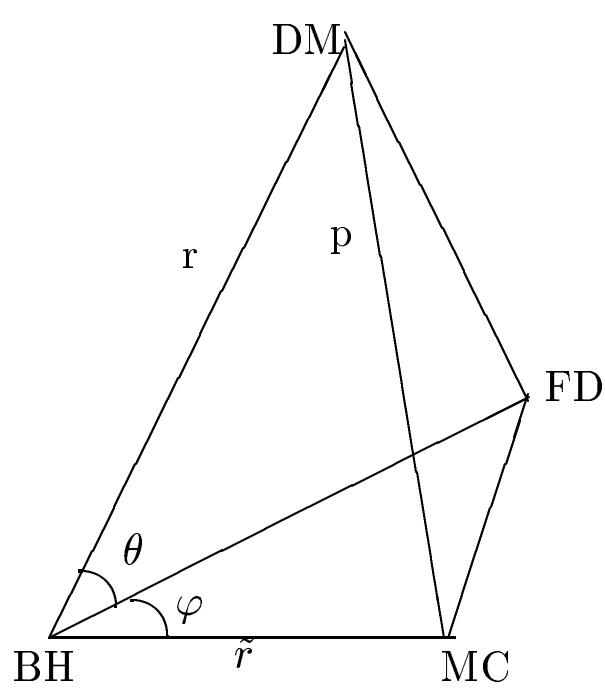

Fig. 4. Geometry for the system BH (BH), DM (DM) particles and molecular clouds (MC). FD stands for the foot (projection) of the DM particle in the disk. The angles BH-FD-DM and MC-FD-DM are both right angles.

gravitational (Coulomb) encounters, we evaluate the random walk integral (Spitzer 1987)

$\Delta v^{2}=\int_{\tilde{r}_{\min }}^{\tilde{r}_{\max }} \int_{0}^{\pi}\left(\frac{2 v p_{0}}{p}\right)^{2} \tilde{r} \mathrm{~d} \tilde{r} \mathrm{~d} \varphi n_{\text {pert }}(\tilde{r}) v \tau_{\text {refill }}(r)$,

where $v$ is the relative velocity of DM particle and molecular clouds at large separation, $p_{0}$ and $p$ are given by

$p_{0}=\frac{G\left(m_{\mathrm{pert}}+m_{\mathrm{DM}}\right)}{v^{2}}, \quad p^{2}=r^{2}+\tilde{r}^{2}-2 r \tilde{r} \cos \theta \cos \varphi$.

In the above equations, we have used the values of $0.1 \mathrm{kpc}$ and $20 \mathrm{kpc}$ for $\tilde{r}_{\min }$ and $\tilde{r}_{\max }$, respectively. We will also assume the following 3D number density distribution equivalent to the surface density for molecular clouds (Güsten \& Mezger 1982)

$n_{\mathrm{pert}}(\tilde{r})=\frac{\sigma_{0}}{2 \tilde{r}} \exp \left(-\frac{\tilde{r}}{r_{0}}\right)$,

where $r_{0}=5 \mathrm{kpc}$ is a scaling distance and $\sigma_{0}$ is a constant denoting the surface density to be chosen a posteriori. Inserting the last expression (32) for the number density into Eq. (30), after integration we get the following equation for the time to refill the loss cone:

$\tau_{\text {refill }}(r)=\frac{v^{3} r_{0}}{2 \pi G^{2}\left(m_{\mathrm{DM}}+m_{\mathrm{pert}}\right)^{2} \sigma_{0} I(r, \theta)}\left(\frac{\Delta v}{v}\right)^{2}$,

with

$\left(\frac{\Delta v}{v}\right)^{2}=\left(\frac{R_{\mathrm{s}}}{r}\right)^{2}=\left(\frac{2 G M_{\mathrm{BH}}}{c^{2} r}\right)^{2}$,

where the function $I(r, \theta)$ is given by the following integral

$I(r, \theta)=\int_{x_{\min }}^{x_{\max }} \mathrm{d} x \frac{\exp (-x)}{\sqrt{x^{4}-2\left(\frac{r}{r_{0}}\right)^{2} \cos (2 \theta) x^{2}+\left(\frac{r}{r_{0}}\right)^{4}}}$, 
In the last integral, $x_{\min }=\tilde{r}_{\min } / r_{0}=0.02$ and $x_{\max }=\tilde{r}_{\max } / r_{0}=$ 4. The function $I(r, \theta)$ tends to a constant for $r \rightarrow 0$ whereas for large values of $r$ it scales as $1 / r^{2}$.

The FB is consumed from inside by the $\mathrm{BH}$ with an accretion rate

$\dot{M}_{\mathrm{BH}}=4 \pi r^{2} \rho v_{\mathrm{ff}}, \quad r<R_{\mathrm{F}}$,

which after using the Pauli degeneracy condition is given by Eq. (21). Thus the BH gains by consuming DM inside the FB and of course the FB loses its DM particles. On the other hand the FB is also fed on the outside by direct collision via the loss cone mechanism. The FB growth rate is given by

$\dot{M}_{\mathrm{FB}}=\int_{R_{\mathrm{F}}}^{R_{\mathrm{DM}}} 4 \pi r^{2} \rho(r) \delta \Omega(r) \tau(r)^{-1} \mathrm{~d} r \quad r>R_{\mathrm{F}}$,

where $R_{\mathrm{F}}$ is the size of the $\mathrm{FB}, R_{\mathrm{DM}}$ is the $\mathrm{DM}$ halo size and $\delta \Omega$ is the fraction of the solid angle subtended by the loss cone and is given by

$\delta \Omega=\pi\left(\frac{R_{\mathrm{s}}}{r}\right)^{2}=\pi\left(\frac{2 G M_{\mathrm{BH}}}{c^{2} r}\right)^{2}$.

The timescale $\tau$ for the FB growth is given by

$\tau=\left\{\begin{array}{lc}t_{\text {dyn }}, & t_{\text {refill }}<t_{\text {dyn }} \\ t_{\text {refill }}, & t_{\text {dyn }}<t_{\text {refill }},\end{array}\right.$

where $t_{\mathrm{dyn}}$ and $t_{\text {refill }}$ are the dynamical (orbital) and loss cone refilling timescales, respectively. As soon as the FB is consumed, the capture radius for DM particles coming from the outside drops rapidly.

In Fig. 5, we plot the dynamical and the refilling timescales as functions of the radius. At small radii, i.e. $r<R_{\mathrm{s}}$, the time $t_{\text {refill }}$ to refill the loss cone is too long for the DM to feed the BH. Near the BH, we use the dynamical timescale to feed the $\mathrm{BH}$. For radii greater than the size of the $\mathrm{FB}$, we will use the slowest of the two timescales to feed the FB. Thus the FB feeds from the outside DM particles via the loss cone mechanism while the $\mathrm{BH}$ feeds from DM inside the FB via the quantum cascade mechanism.

In Fig. 6, we plot the mass flow as a function of the radius from the center. To this end, we fix the mass of the FB to $3 \times$ $10^{6} M_{\odot}$ and use a fermion mass of $m_{\mathrm{f}} \sim 12 \mathrm{keV} / c^{2}$. Using the $\mathrm{BH}$ accretion rate Eq. (21), the accretion rate becomes $\dot{M} \sim$ $10^{6} M_{\odot} \mathrm{yr}^{-1}$ for a fermion mass of $m_{\mathrm{f}}=12 \mathrm{keV} / c^{2}$ and a BH mass of $3 \times 10^{6} M_{\odot}$. For fermions of mass $450 \mathrm{keV} / c^{2}$ and a BH mass of $3 \times 10^{3} M_{\odot}$, the accretion rate is $\dot{M} \sim 10^{4} M_{\odot} \mathrm{yr}^{-1}$. It is seen than the $\mathrm{BH}$ accretion rate is independent on the radius and drops significantly when the size of the FB is reached. Thus the $\mathrm{BH}$ grows much faster from the inner DM particles than the FB from the outer DM particles. After the $\mathrm{BH}$ has reached a mass equal to that of the $\mathrm{FB}$, i.e. $10^{3-4} M_{\odot}$, its growth is controlled by Eddington baryonic matter accretion.

In Fig. 7, we plot the BH mass as a function of time for different values of the fermion mass. The $\mathrm{BH}$ mass - time dependence is given by Eq. (26). It is shown that for fermion masses lower than about $1 \mathrm{keV}$, it would take more than $10^{10}$ years for

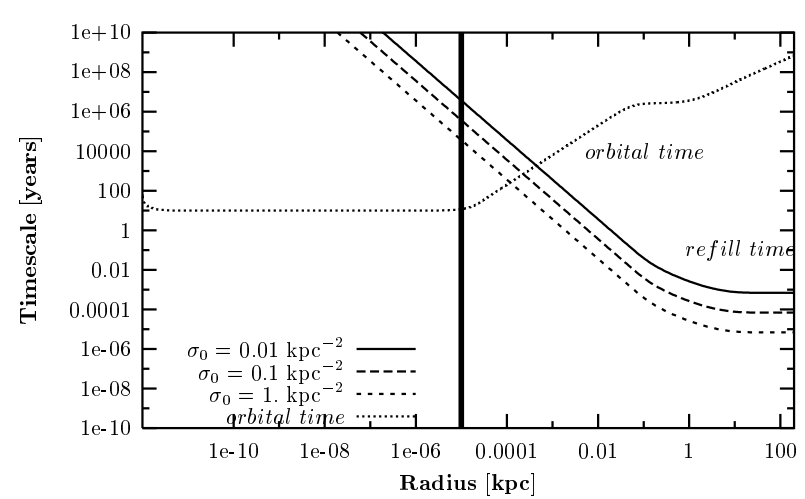

Fig. 5. The refilling and orbital timescales plotted as a function of the radius from the center of the DM halo. In this plot, the mass of the FB is $3 \times 10^{6} M_{\odot}$ and the corresponding fermion mass is $12 \mathrm{keV} / c^{2}$. For small $r$, the time to refill the loss cone is much greater than the age of the Universe. For the growth of the $\mathrm{BH}$, we use the orbital time scale and the $\mathrm{BH}$ feeds on DM from inside the FB. For the DM inside the FB to be fully consumed by the $\mathrm{BH}$, we introduce a quantum cascade effect which is a result of the existence of a high degeneracy pressure that pushes high angular momentum particles to the inner Fermi surface. The particles at the inner Fermi surface in 6D phase space will then be consumed by the $\mathrm{BH}$. This process continues until the $\mathrm{BH}$ has consumed the entire FB. The BH then grows by Eddington-limited baryonic matter accretion. The FB is fed from DM from outer orbits but its accretion rate is much lower than that of the $\mathrm{BH}$ inside the FB as seen in the next figure. The vertical thick line shows the size of the FB.

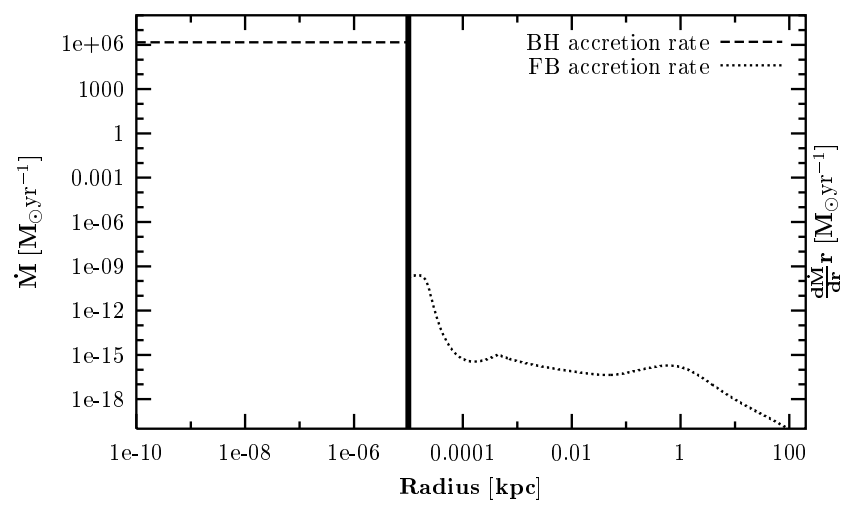

Fig. 6. The mass accretion flow as a function of distance from the center. For a given mass of the $\mathrm{BH}$, the accretion rate is independent of the radius until the Bondi radius where the $\mathrm{BH}$ dominates the gravitational potential of the fermions. As the $\mathrm{BH}$ mass approaches that of the Fermion ball, the $\mathrm{BH}$ accretion rate becomes constant until the radius of the FB and as the size of the FB is reached, the DM accretion rate onto the FB drops dramatically. Thus the growth rate of the FB is much lower than that of the $\mathrm{BH}$ which grows by feeding on DM from inside the FB. Similarly to the last plot, we have used here a FB mass of $3 \times 10^{6} M_{\odot}$. The left side of the plot shows the $\mathrm{BH}$ accretion rate and the $\mathrm{FB}$ consumption rate is shown on the right side of the figure.

the BH to grow to $10^{3-4} M_{\odot}$. However, due to the Pauli principle used in the derivation of Eq. (26), if the fermion mass is constrained in the mass range between about $1 \mathrm{keV}$ and $1 \mathrm{MeV}$, a seed $\mathrm{BH}$ of $5 M_{\odot}$ can grow to a $10^{3-4} M_{\odot} \mathrm{BH}$ in about $10^{7-8}$ years, which would then grow by Eddington-limited baryonic accretion to $10^{9} M_{\odot}$ at a redshift of $z \approx 6.41$ (see next 


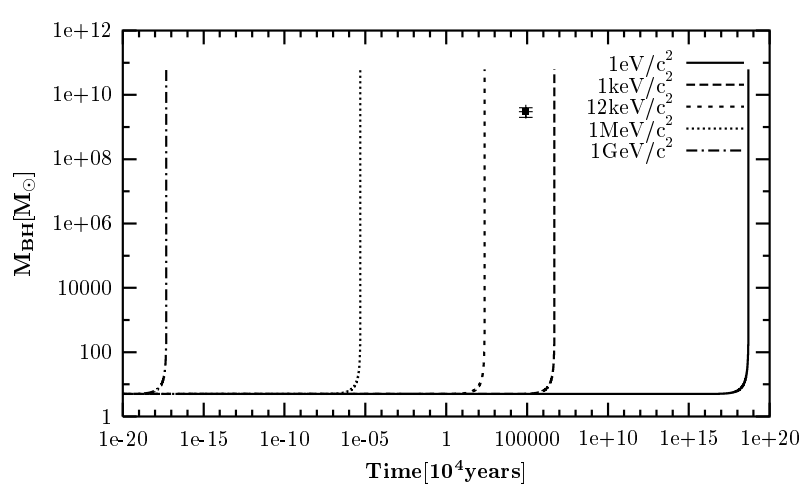

Fig. 7. The black hole mass growth from only DM accretion. The fermion mass is varied as shown on the plot. It can be seen that the $\mathrm{BH}$ mass can grow to $10^{3-4} M_{\odot}$ in about $10^{7-8}$ years if the fermion mass is in the range between about $1 \mathrm{keV}$ and $1 \mathrm{MeV}$. For fermion masses $m_{\mathrm{f}} \lesssim 1 \mathrm{keV} / c^{2}$, the formation of a $\mathrm{BH}$ of $10^{3} M_{\odot}$ would require a timescale much greater than than a Hubble time. On the other hand, for fermion masses $m_{\mathrm{f}} \gtrsim 1 \mathrm{MeV} / c^{2}$, a $10^{3} M_{\odot} \mathrm{BH}$ would have formed in less than than one year after the beginning of the Universe. Thus the degeneracy pressure of fermions allows for the capture of a large amount of mass if the fermion mass is constrained in a range between about $1 \mathrm{keV}$ and $1 \mathrm{MeV}$. The data point shown at $t=8.47 \times 10^{8}$ years is the recent detection of a $\mathrm{BH}$ mass of $3 \times 10^{9} M_{\odot} \mathrm{BH}$ in the quasar SDSS SDSS J114816.64+525150.3.

subsection). Fermion masses greater than about $1 \mathrm{MeV}$ would make the $\mathrm{BH}$ grow much faster and the $\mathrm{BH}$ could be as large as $10^{3-4} M_{\odot}$ in less than about one year after the beginning of the Universe.

In the next section, we will discuss the Eddington baryonic matter accretion and discuss the plots of $\mathrm{BH}$ growth from both $\mathrm{DM}$ and Eddington baryonic matter accretion.

\subsection{Eddington limited baryonic accretion}

The details of Eddington-limited baryonic accretion have been studied by many authors, here we refer the reader to Wang \& Biermann (1998) and provide just the main formulae:

$\frac{\mathrm{d} M_{\mathrm{BH}}}{\mathrm{d} t}=\frac{M_{\mathrm{BH}}}{\tau_{\mathrm{EDD}}}$,

where $\tau_{\mathrm{EDD}}=10^{7.69}$ years $\times\left(\frac{\epsilon}{0.1}\right)$ is the corresponding time scale, $\epsilon$ being the efficiency. This value for $\tau_{\text {EDD }}$ has been chosen to fit the exponential growth of BHs given by Fig. 1 of Wang \& Biermann (1998). The BH mass is found to grow in this case as

$M_{\mathrm{BH}}(t)=M_{\mathrm{BH}}^{i} \exp \left(\frac{t-t^{i}}{\tau_{\mathrm{EDD}}}\right)$,

and the corresponding Eddington luminosity for an efficiency $\epsilon$ of 0.1 is

$L=\epsilon \dot{M}_{\mathrm{BH}} c^{2}=0.1 \frac{M_{\mathrm{BH}}}{\tau_{\mathrm{EDD}}} c^{2}$.

In Figs. 8 and 9, we present the growth of supermassive BHs accreting DM and baryonic matter. It is seen from the plot that a $\mathrm{BH}$ grows faster in the beginning accreting DM rather than baryonic matter. In Fig. 8, the degenerate FB has a mass of

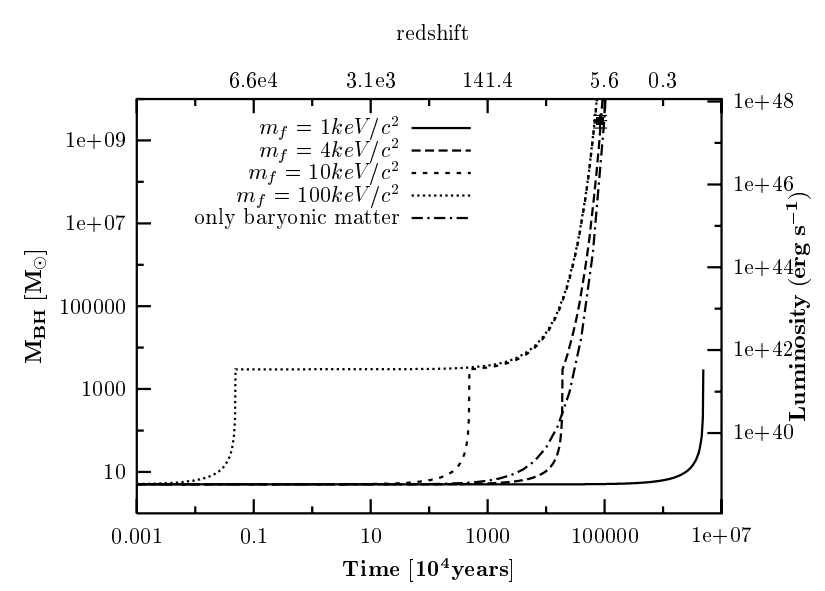

Fig. 8. The mass $M_{\mathrm{BH}}$ of the $\mathrm{BH}$ is plotted as a function of time $t$ for $\mathrm{DM}$ and Eddington-limited baryonic matter accretion. It is seen from this plot that DM dominates the $\mathrm{BH}$ growth at early times. The mass of fermions is varied as shown on the plot. The total mass of the degenerate $\mathrm{FB}$ has a mass of $3 \times 10^{3} M_{\odot}$. We find that fermion masses of $m_{\mathrm{f}} \gtrsim 4 \mathrm{keV} / c^{2}$ suffice for stellar seed BHs to grow to about $10^{3} M_{\odot}$ which then grow by Eddington-limited baryonic matter accretion to about $3 \times 10^{9} M_{\odot}$. The obtained constraint on the fermion mass covers the range of dark matter masses discussed earlier. The data point shown at $z=6.41$ corresponds to the $\mathrm{BH}$ mass of $\sim 3 \times 10^{9} M_{\odot}$ in the quasar SDSS J114816.64+525150.3.

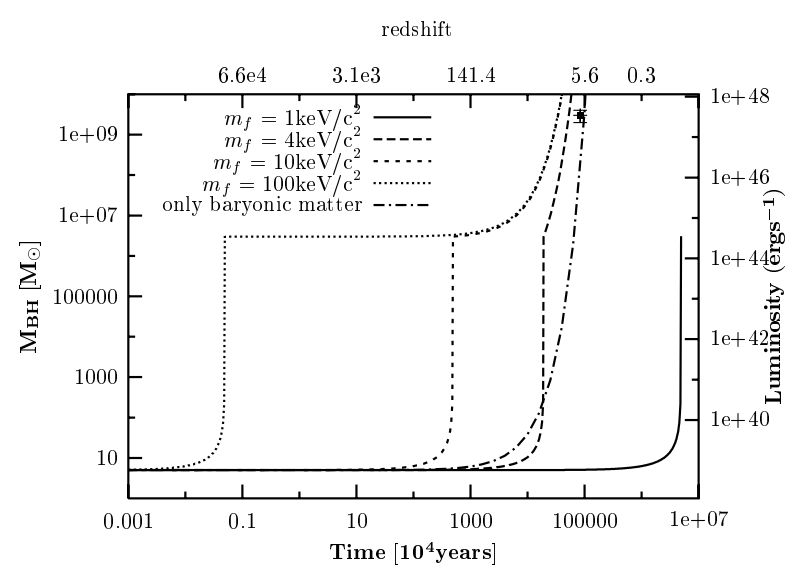

Fig. 9. The same as in the last figure but for a degenerate FB mass of $3 \times 10^{6} M_{\odot}$.

$3 \times 10^{3} M_{\odot}$ whereas we use a FB mass of $3 \times 10^{6} M_{\odot}$ in Fig. 9 . The data point shown in Figs. 8 and 9 at $t=8.4 \times 10^{8}$ years is the recent detection of a supermassive $\mathrm{BH}$ of mass of $M_{\mathrm{BH}} \sim$ $3 \times 10^{9} M_{\odot}$ in the quasar SDSS J114816.64+5251 at a redshift of $z=6.41$ (Willott et al. 2003; Fan et al. 2001).

It is seen from Figs. 8 and 9 that only baryonic matter accretion cannot fit this data point and the curve for the growth of the $\mathrm{BH}$ due to baryonic matter lies on the right side of the data point. Using Eq. (41) the total mass of the $\mathrm{BH}$ from Eddington-limited baryonic accretion at $t=8.4 \times 10^{8}$ years i.e. $z=6.41$ is $1.4 \times 10^{8} M_{\odot}$ which is about ten times less than the inferred BH mass of $3 \times 10^{9} M_{\odot}$, clearly outside the error range. The growth of a seed $\mathrm{BH}$ from $5 M_{\odot}$ to $3 \times 10^{9} M_{\odot}$ is accomplished in two steps: First, the BH completely consumes the degenerate Fermi core with masses between $3 \times 10^{3} M_{\odot}$ and 


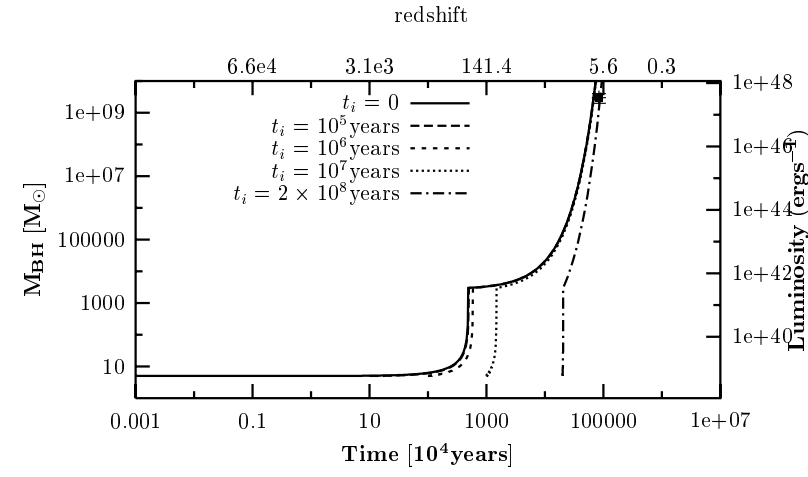

Fig. 10. The BH growth dependence on the starting time of accretion. We find that starting DM accretion at a time of $2 \times 10^{8}$ years would fit the recent discovery of a $3 \times 10^{9} M_{\odot} \mathrm{BH}$ in the quasar SDSS J1148 +5251 at a redshift of $z=6.41$, which is the most distant known quasar, observed only 840 million years after the beginning of the Universe (Willot et al. 2003). The fermion mass used in this plot is $m_{\mathrm{f}}=10 \mathrm{keV} / c^{2}$. However, any higher fermion mass would more sharply and rapidly raise the mass of a seed $\mathrm{BH}$ from $5 M_{\odot}$ to $\sim 10^{3} M_{\odot}$.

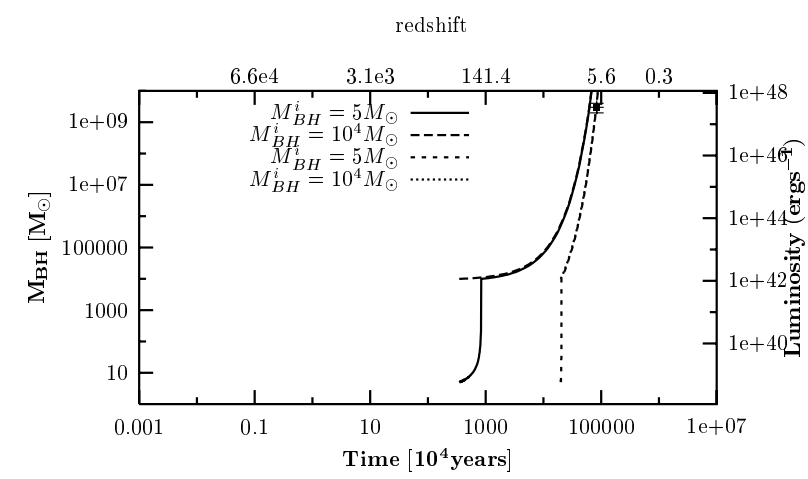

Fig. 11. We investigate how the $\mathrm{BH}$ growth depends on the mass of the seed BH. To fit the data point of mass $M \sim 3 \times 10^{9} M_{\odot}$ at $z \approx 6.41$, the seed BH cannot be greater than $10^{4} M_{\odot}$ for a FB of $10^{4} M_{\odot}$ and the accretion starting time should be about $z \approx 17$. In this plot, the fermion mass is fixed to $m_{\mathrm{f}} \approx 10 \mathrm{keV} / c^{2}$. Thus, any other mechanism that could create massive seed BHs would generate the same growth.

$3 \times 10^{6} M_{\odot}$. In the second step, the $10^{3}-10^{6} M_{\odot}$ BH feeds on baryonic matter accretion to reach the mass of $3 \times 10^{9} M_{\odot}$ at a redshift of $z \sim 6.41$.

In Fig. 10, we investigate how the growth of the mass of the $\mathrm{BH}$ depends on the accretion starting time. To this effect, we fix the total mass of the FB to $3 \times 10^{3} M_{\odot}$ and the fermion mass to $m_{\mathrm{f}}=10 \mathrm{keV} / c^{2}$ and vary the initial time when the DM accretion starts as shown on the graph. We find that the data point of $3 \times 10^{9} M_{\odot}$ at $z \sim 6.41$ is well fitted for an accretion starting time of $t \sim 2 \times 10^{8}$ years for any fermion greater than $m_{\mathrm{f}} \gtrsim 10 \mathrm{keV} / c^{2}$. Here we also note that Eddington baryonic matter accretion could also allow the seed $\mathrm{BH}$ to grow to a $3 \times 10^{9} M_{\odot} \mathrm{BH}$ if the efficiency is $\epsilon=1.1 \times 10^{-2}$. The accretion starting time of $t \sim 2 \times 10^{8}$ years corresponds to a redshift of reionization of $z \approx 17$ as obtained by WMAP observations (Krauss 2003).

Finally in Fig. 11, we investigate how the growth of the $\mathrm{BH}$ is affected by the mass of the seed $\mathrm{BH}$ at different starting times of accretion. In order to constrain the mass of the seed $\mathrm{BH}$, we vary the mass of the FB from $10^{3} M_{\odot}$ to $10^{6} M_{\odot}$ and also the mass of the seed BH from $5 M_{\odot}$ to $10^{4} M_{\odot}$ at initial times of $t^{i}=3.5 \times 10^{6}$ and $2 \times 10^{8}$ years. These values correspond to the earliest and latest plausible times for the formation of the first stellar seed BHs in order to grow into supermassive BHs at redshift $z \approx 6.41$. From recent WMAP observations (Spergel et al. 2003), BHs of masses $\sim 10^{5} M_{\odot}$ might have formed at a redshift $z \geq 20$. In Fig. 11, we have plotted only the limiting curves which show that the mass of the FB should be $M_{\mathrm{F}} \leq$ $10^{4} M_{\odot}$ and the mass of the seed $\mathrm{BH}$ cannot be greater than about $10^{4} M_{\odot}$. The best fit is obtained for a BH formation time a reionization time of $t^{i} \sim 2 \times 10^{8}$ years, i.e. $z \approx 17$.

It is interesting to estimate the maximum luminosity attainable by an accreting BH of this mass. This is usually considered to be the Eddington luminosity at which the outward radiation pressure equals the inward gravitational attraction. Using Eq. (42), the luminosity of a $3 \times 10^{9} M_{\odot} \mathrm{BH}$ is of the order of a few $10^{47} \mathrm{erg} \mathrm{s}^{-1}$ and the corresponding accretion rate is about $65 M_{\odot} /$ yr. However, such high luminosity values (Fan et al. 2001) would be obtained only for a standard efficiency of $\epsilon=0.1$ which as seen from Figs. 8 and 9 does not fit the data point for only baryonic matter accretion.

\subsection{BH masses from energy conservation arguments}

A gravitational system in which molecular clouds provide the interaction path between DM particles and feeding the $\mathrm{BH}$ leads to a strong limit on the maximum mass of the $\mathrm{BH}$. For an initial distribution of $\mathrm{DM}$ in the halo without a $\mathrm{BH}$, the total energy of the system is given by

$E_{\mathrm{i}}=\frac{-3 G M_{\mathrm{DM}}^{2}}{5 R_{\mathrm{DM}}}$,

where $M_{\mathrm{DM}}, R_{\mathrm{DM}}$ stand for the total mass of the DM halo and its size, respectively. After the $\mathrm{BH}$ is formed at the DM halo center, its total energy is given by

$E_{\mathrm{f}}=\frac{-3 G M_{\mathrm{BH}}^{2}}{5 R_{\mathrm{S}}}$,

with $R_{\mathrm{S}}$ being the Schwarzschild radius

$R_{\mathrm{s}}=\frac{2 G M_{\mathrm{BH}}}{c^{2}}$.

Equating $E_{\mathrm{f}}$ to $E_{\mathrm{i}}$ as a limit, we arrive at the following constraint

$\frac{M_{\mathrm{DM}}}{M_{\mathrm{BH}}}=\left(\frac{R_{\mathrm{DM}}}{R_{\mathrm{S}}}\right)^{1 / 2}$,

from which we derive the $\mathrm{BH}$ mass limit

$M_{\mathrm{BH}}^{\max }=\frac{2 G M_{\mathrm{DM}}^{2}}{c^{2} R_{\mathrm{DM}}}$.

Assuming an isothermal gas sphere distribution for the DM halo, i.e. $M_{\mathrm{DM}}=2 \sigma^{2} R_{\mathrm{DM}} / G$, with $\sigma$ being the $\mathrm{DM}$ velocity dispersion, we arrive at the final expression for the $\mathrm{BH}$ mass

$M_{\mathrm{BH}}^{\max }=\frac{8 \sigma^{4} R_{\mathrm{DM}}}{c^{2} G}$, 
or

$M_{\mathrm{BH}}^{\max }=4\left(\frac{\sigma}{c}\right)^{2} M_{\mathrm{DM}}$

The last two equations constrain the properties of the DM matter halo to the $\mathrm{BH}$ and could be considered as the theoretical interpretation of the $M_{\mathrm{BH}}-\sigma$ observed correlation in elliptical galaxies. Here, we note that $\sigma$ stands for the velocity dispersion of DM. Using the above equations, the $\mathrm{BH}$ mass limit $M_{\mathrm{BH}}^{\max }$ is obtained to be $\sim 10^{2} M_{\odot}, \sim 10^{6} M_{\odot}$, and $\sim 10^{9} M_{\odot}$ for dwarf galaxies, the Milky Way and M87, respectively. The corresponding DM halos have masses of $\sim 4 \times 10^{9} M_{\odot}, \sim 3 \times 10^{12} M_{\odot}$ and $\sim 4 \times 10^{14} M_{\odot}$. In order to get the above estimates for the maximum $\mathrm{BH}$ mass, we have used a DM halo size of $R_{\mathrm{DM}} \sim 9 \mathrm{kpc}$ for dwarf galaxies, $R_{\mathrm{DM}} \sim 200 \mathrm{kpc}$ for the Milky Way and the size of M87 is assumed to be $\sim 5 \mathrm{Mpc}$.

\section{Discussion and conclusion}

In this paper, we have investigated the growth of a stellar seed $\mathrm{BH}$ immersed at the center of DM halos with degenerate FBs of mass from $\sim 10^{3} M_{\odot}$ to $\sim 10^{6} M_{\odot}$. Using the Pauli exclusion principle, we have established that the $\mathrm{BH}$ accretion rate strongly depends on the mass $m_{\mathrm{f}}$ of the fermions as $\dot{M}_{\mathrm{BH}} \sim m_{\mathrm{f}}^{4} M_{\mathrm{BH}}^{2}$ and thus establish for the first time the relationship between $\mathrm{BH}$ growth and fermionic DM. We have shown that in order to fit the DM distribution in the Galaxy with such degenerate cores, the DM particles should be in the $12 \mathrm{keV} / c^{2} \lesssim m_{\mathrm{f}} \lesssim 450 \mathrm{keV} / c^{2}$ mass range. We have shown that such DM masses could be used to fit the distribution of DM in dwarf galaxies. FBs of masses $10^{3-6} M_{\odot}$ could only exist in such galaxies where the density drops off as $1 / r^{2}$ at large distances. Dwarf galaxies as well as cluster of galaxies do not host FBs as their data can only be fitted by a non-degenerate Fermi-Dirac distribution of the King type. We have argued that the merging of dwarf galaxies would lead to the formation of galaxies with degenerate FBs. The wideness of the fermion mass in the $\mathrm{keV}$ range is due to the $\mathrm{FB}$ mass range from $10^{3}$ to $10^{6} M_{\odot}$ that we have adopted in this paper. Our main assumption is the use of fermions as DM candidates in galaxies. However, if one uses bosons instead of fermions, the range of DM particle masses would of course differ from the one obtained in this paper. The range of fermions used in our paper is in conflict with the Lee-Weinberg lower limit of $\sim 2 \mathrm{GeV} / c^{2}$ on the fermion mass (Lee \& Weinberg 1977). This is due to the fact that the derivation of the Lee-Weinberg limit assumes a freezout from equilibrium distributions. Our simple model uses the chemical potential which allows for non equilibrium distributions and this might modify the Lee-Weinberg argument; this remains to be demonstrated.

If we use heavy fermions with masses of about $1 \mathrm{GeV} / c^{2}$, then according to Eq. (24) the maximum mass allowed for the degenerate Fermi core would only be of about $1 M_{\odot}$, which is not enough to grow a stellar mass $\mathrm{BH}$ to $10^{3} M_{\odot}$ in about $10^{8}$ years. On the other hand, very light fermions i.e. $m_{\mathrm{f}} \lesssim$ $12 \mathrm{keV} / \mathrm{c}^{2}$ would generate very massive degenerate FBs of masses greater than $10^{6} M_{\odot}$ and the $\mathrm{BH}$ would grow to $10^{9} M_{\odot}$ in a very short time, i.e $z \ll 6.41$.
The growth of seed BHs from DM accretion is investigated using the quantum cascade mechanism upon which low angular momentum DM particles at the inner Fermi surface are first consumed by the $\mathrm{BH}$ and then due to a high degeneracy pressure, higher angular momentum particles are pushed inwards and the process continues until the entire degenerate FB is consumed by the $\mathrm{BH}$. Moreover, molecular clouds have been used as perturbers of DM particle orbits outside the FB and we have shown that the $\mathrm{BH}$ grows faster than the FB. After the $\mathrm{BH}$ has consumed the entire FB, it then grows by Eddington-limited baryonic accretion to higher masses of $\sim 10^{9} M_{\odot}$ at redshifts $z \sim 6.41$. We also point out that molecular clouds of mass $10^{10} M_{\odot}$ have also been detected in the host galaxy of the same quasar at a redshift of $z \sim 6.41$ (Walter et al. 2003).

We have also constrained the possible starting time of accretion, i.e. the time of $\mathrm{BH}$ seed formation. From our analysis, the mass of a $3 \times 10^{9} M_{\odot} \mathrm{BH}$ in the quasar SDSS sJ114816.64+525150.3 at a redshift of $z=6.41$ can be fitted exactly if the accretion process starts at a time of about $2 \times 10^{8}$ years, which corresponds to the reionization time.

The seed BH mass is found to be in the range from a few solar masses up to an upper limit of $\sim 10^{4} M_{\odot}$. For a seed BH mass of $10^{3-4} M_{\odot}$, Eddington baryonic matter accretion would be enough to cause the seed $\mathrm{BH}$ to grow into a supermassive $\mathrm{BH}$ of $3 \times 10^{9} M_{\odot}$ mass. The data point at a redshift of $z=6.41$ can be fitted by only Eddington baryonic matter accretion with an efficiency of $\epsilon \sim 0.01$.

Our model provides a method to find the DM particles mass. If it is found that there is a clear lower mass cut of $10^{3}$ to $10^{6} M_{\odot}$ in the distribution of $\mathrm{BH}$ masses, then this mass can be used for the mass of the FB to find the corresponding mass $m_{\mathrm{f}}$ of the fermions which will be in the range of $12 \mathrm{keV}$ to $450 \mathrm{keV}$. If on the other hand the $\mathrm{BH}$ mass distribution is a continuous function, then our model of BH growth with DM will probably be ruled out.

The postulated DM particles in this paper were nonrelativistic at the decoupling time and are usually called cold dark matter particles (CDM). The latter have to be neutral, stable or quasi-stable and have to weakly interact with ordinary matter. As mentioned in Sect. 2.1, these particles could be axions which have been investigated by DAMA/NAI (Bernabei et al. 2001). The heavier particles of mass above $1 \mathrm{GeV} / c^{2}$ could also be of the class of DM candidates named WIMPS (Weakly Interactive Massive particles). However, in the standard model of particle physics, CDM cannot be suitable candidates for particles. Thus, a new window beyond the standard model of particle physics has to accommodate these particles for our model of BH growth to work. The DAMA/NAI experiment (see Bernabei et al. 2004, for a review) which aims at the verification of the presence of DM particles in the Galactic halo, will be able to confirm whether GeV WIMPS or axions of masses of 12 to $450 \mathrm{keV}$ could exist in nature.

Observations have shown that the masses of supermassive BHs at galactic centers correlate with the masses of the host bulges, i.e. $M_{\mathrm{BH}} \approx 0.002 M_{\text {bulge }}$ (Haering \& Rix 2004). This result is obtained in our model as long as the Eddington limited accretion dominates the final growth of the $\mathrm{BH}$. This happens for BH masses of more than about $10^{5} M_{\odot}$ (Wang et al. 2000). 
Mergers of dwarf galaxies as well as the spinning of BHs would play an important role in the growth of the BHs. The consideration of these two effects will be the subject of further investigations. In addition, it would be of great interest to study the growth of BHs from boson DM particles. Although it has been shown that bosons could provide a good fit to the rotation curves in dwarf galaxies, it is not yet clear whether an analogous mechanism could work in galaxies with a $1 / r^{2}$ density fall off.

While it takes only $8.4 \times 10^{8}$ years to grow supermassive BHs in most distant quasars, the Galactic center might have grown to its current mass of $\sim 10^{6} M_{\odot}$ with only DM accretion in a Hubble time. In the following paper, we will address the growth of the Galactic center BH.

Acknowledgements. We thank Yiping Wang for providing us with the data file for baryonic matter growth. We are also grateful to Heino Falcke and Rainer Spurzem for useful discussions. F.M. research is supported by the Alexander von Humboldt Foundation. Work with P.L.B. has been supported through the AUGER theory and membership grant 05 CU1ERA/3 through DESY/BMF (Germany). Further support for the work with PLB has come from the DFG, DAAD, Humboldt Foundation (all Germany), grant 2000/066950 from FAPESP (Brasil) through G. Medina-Tanco, a grant from KOSEF (Korea) through H. Kang and D. Ryu, a grant from ARC (Australia) through R. J. Protheroe, and European INTAS/ Erasmus/ Sokrates/Phare grants with partners V. Berezinsky, L. Gergely, M. Ostrowski, K. Petrovay, A. Petrusel, M. V. Rusu and S. Vidrih.

\section{References}

Bahcall, J. N., \& Wolf, R. A. 1976, ApJ, 209, 214

Baltz, E. A. [arXiv:astro-ph/0412170]

Barkana, R., Haiman, Z., \& Ostriker, J. P. 2001, ApJ, 558, 482

Bernabei, B., Belli, P., Cerulli, R., et al. 2001, Phys. Lett. B, 515, 6

Bernabei, B., Belli, P., Cappella, F., et al. 2004, Int. J. Mod. Phys. D, 13,2127

Bertone, G., \& Silk, G. 2002, MNRAS, 337, 98

Bertone, G., Hooper, D., \& Silk, J. 2005, Phys. Rep., 405, 279

Bilić, N., Munyaneza, F., Tupper, G. B., \& Viollier, R. D. 2002a, Prog. Part. Nucl. Phys., 48, 291

Bilić, N., Munyaneza, F., Tupper, G. B., \& Viollier, R. D. 2002b, in Proc. of the 4th International Conference DARK 2002 of dark matter in Astro - Particle Physics, ed. Klapdor-Kleingrothaus, \& R. D. Viollier, 46

Bilić, N., Munyaneza, F., \& Viollier, R. D. 1999, Phys. Rev. D, 59, 024003

Carignan, C., \& Purton, C. 1998, ApJ, 506, 125

Carilli, C. L., Solomon, P., Vanden Bout, P., et al. 2005, ApJ, 618, 586

Coleman Miller, M. 2003 [arXiv: astro-ph/0306173]

Cowsik, R., \& McClelland, J. 1973, ApJ, 180, 7

Duschl, W. J., \& Strittmatter, P. A. 2004 [arXiv: astro-ph/0401010]

Duncan, M. J., \& Shapiro, S. L. 1982, ApJ, 253, 921

Duncan, M. J., \& Wheeler, J. C. 1980, ApJ, 237, L27

Fan, X., Narayanan, V. K., Lupton, R. H., et al. 2001, AJ, 122, 2833

Faber, S. M., Tremaine, S., Ajhar, E. A., et al. 1997, AJ, 114, 1771

Ferrarese, L. 2002, ApJ, 578,90

Ferrarese, L., \& Merritt, D. 2000, ApJ, 539, L9

Frank, J., \& Rees, M. J. 1976, MNRAS, 176, 633

Gebhardt, K., Richstone, D., Kormendy, J., et al. 2000, AJ, 119, 1157

Gondolo, P., \& Silk, J. 1999, Phys. Rev. Lett., 83, 1719
Gopal-Krishna, Biermann, P. L., \& Wiita Paul, J. 2003, ApJ, 594, L103

Gopal-Krishna, Biermann, P. L., \& Wiita Paul, J. 2004, ApJ, 603, L9

Goto, T., \& Yamaguchi, M. 1992, Phys. Lett. B, 276, 123

Güsten, R., \& Mezger, P. G. 1982, Vistas in Astron., 26, 1982

Haering, N., \& Rix, H. 2004, ApJ, 604, L89

Hills, J. G. 1975, Nature, 254, 295

Julian, W. 1967, ApJ, 148, 175

Krauss, L. M. 2003, ApJ, 596, L1

King, A. 1966, AJ, 71, 64

Kravtsov, A. V., Klypin, A. A., Bullock, J. S., \& Primack, J. R. 1998, ApJ, 502, 48

Lee, B. W., \& Weinberg, S. 1977, Phys. Rev. Lett., 39, 165

Lightman, A. P., \& Shapiro, S. L. 1978, Rev. Mod. Phys., 50, 437

Lynden-Bell, D. 1969, Nature, 223, 690

Lyth, D. H. 2000, Phys. Lett. B, 488, 417

MacMillan, J. D., \& Henriksen, R. N. 2002, ApJ, 569, 83

Macchetto, D., Marconi, A., Axon, D. J., et al. 1997, ApJ, 489, 579

Magorrian, J., Tremaine, S., Richstone, D., et al. 1998, AJ, 115, 2285

Markov, M. A. 1964, Phys. Lett., 10, 22

Merritt, D. 2004, Phys. Rev. Lett., 92, 201304

Merritt, D., Milosavljevic, M., Verde, L., \& Jimenez, R. 2002, Phys. Rev. Lett., 88, 1301

Munyaneza, F., \& Viollier, R. D. 2002, ApJ, 564, 274

Munyaneza, F., Tsiklauri, D., \& Viollier, R. D. 1998, ApJ, 509, L105

Munyaneza, F., Tsiklauri, D., \& Viollier, R. D. 1999, ApJ, 526, 744

Navarro, J. F., Frenk, C. S., \& White, S. D. M. 1997, ApJ, 490, 493

Ostriker, J. P. 2000, Phys. Rev. Lett., 84, 23

Peebles, P. J. E. 1972, Gen. Rel. Grav., 3, 6

Podsiadlowski, Ph., Rappaport, S., \& Hau, Z. 2003, MNRAS, 341, 385

Portegies Zwart, S. F., \& McMillan, S. L. W. 2002, ApJ, 576, 899

Read, J. I., \& Gilmore, G. 2003, MNRAS, 339, 949

Rees, M. J. 1984, ARA\&A, 22, 471

Ruffini, R. 1980, Lett. Nuovo Cim., 29, 161

Quinlan, G. D., Hernquist, L., \& Sigurdsson, S. 1995, ApJ, 440, 554

Schödel, R., Ott, T., Genzel, R., et al. 2002, Nature, 419, 694

Schunk, F. E., \& Liddle, A. R. 1977, Phys. Lett. B, 404, 25

Schunk, F. E., \& Mielke, E. W. 2003, Class. Quant. Grav., 20, R301

Silk, J., \& Rees, M. J. 1998, A\&A, 331, L1

Spergel, D. N., \& Steinhardt, P. J. 2000, Phys. Rev. Lett., 84, 3760

Spergel, D. N., Verde, L., Peiris, H. V., et al. 2003, ApJS, 148, 175

Shapiro, S. L. 1985, in Dynamics of star clusters, ed. J. Goodman, \& P. Hut (Dordrecht: Reidel), IAU Symp., 113, 373

Spitzer, L. 1987, Dynamical evolution of globular clusters (Princeton: Princeton Univ. Press)

Spitzer, L., \& Schwarzschild, M. 1951, ApJ, 114, 3856

Spitzer, L., \& Schwarzschild, M, 1953, ApJ, 118, 106

Torres, D. F., Capozziello, S., \& Lambiase, G. 2000, Phys. Rev. D, 62, 104012

Tsiklauri, D., \& Viollier, R. D. 1998, ApJ, 500, 591

Tyler, C., Janus, B., \& Santos-Noble, D. 2003 [arXiv:astro-ph/0309008]

van der Marel, R. P. 2003, Carnegie Observatories Astrophysics series, Vol. 1: Coevolution of Black Holes and Galaxies, ed. L. C. Ho, in press [arXiv: astro-ph/0302101]

van Albada, T. S., Bahcall, J. N., Begeman, K., \& Sancisi, R. 1985, ApJ, 295, 305

Viollier, R. D. 1994, Prog. Part. Nucl. Phys., 32, 51

Walter, F., Bertoldi, F., Carilli, C., et al. 2003, Nature, 424, 406

Wang, Y., \& Biermann, P. L. 1998, A\&A, 334, 87

Wang, Y., Biermann, P. L., \& Wandel, A. 2000, A\&A, 361, 550

Willott, C. J., McLure, R. J., \& Jarvis, M. 2003, ApJ, 587, L15

Wilkinson, M. I., \& Evans, N. W. 1999, MNRAS, 310, 645

Young, P. 1980, ApJ, 242, 1232

Zhao, H., Haehnelt, M. G., \& Rees, M. J. 2002, New Astron., 7, 385 